\title{
Designing a duo of material and digital artifacts: the pascaline and Cabri Elem e-books in primary school mathematics
}

\author{
Michela Maschietto \\ Dipartimento di Educazione e Scienze Umane \\ Università di Modena e Reggio Emilia \\ Via Allegri, 9 \\ 42100 Reggio Emilia (IT)
}

Sophie Soury-Lavergne

S2HEP

Institut Français de l'Education

Ecole Normale Supérieure de Lyon

19 allée de Fontenay BP 17424

69347 Lyon Cedex 07 (FR)

\begin{abstract}
This paper focuses on a duo of artifacts, constituted by a physical artifact and its digital counterpart. It deals with the theoretically and empirically underpinned design process of the digital artifact, the e-pascaline developed with Cabri Elem technology, in reference to a physical artifact, the pascaline. The theoretical frameworks of the instrumental approach and the theory of semiotic mediation together with the analysis of teaching experiments with the pascaline support the design in terms of continuity and discontinuity between the two artifacts. The components of the digital artifact were chosen from among the components of the physical artifact that are the object of instrumental genesis by the students and that are analyzed as having a semiotic potential that contributes to didactical aims. Components instrumented by students which had inadequate semiotic potential were eliminated. With the resulting duo, each artifact adds value to the use of the other artifact for mathematical learning.
\end{abstract}

\section{Introduction}

As suggested by Pepin, Gueudet, and Trouche (2013) in the introduction to this issue of ZDM, the number of available resources for teaching seems to be continually increasing. Two different types of resource can be distinguished: physical resources (referred to as 
classical resources by WG4 at the ICMI Symposium in Rome $2008^{1}$ ) and digital resources. Much research already exists about the utility of each kind of resource for the learning process. With regard to physical resources, the role of gesture and sensory-motor experiences in bodily and kinesthetic activities for mathematical conceptualization has been established in different research fields: in cognitive sciences (Lakoff and Núñez 2000), in developmental psychology (Kalénine et al. 2011), and in mathematics education (Edwards et al. 2009)

With regard to digital technologies for mathematics learning, a similar research trend exists (see the Kaput Center Projects or Hoyles and Lagrange 2010). However, very little research focuses on the combined use of the two kinds of resource, physical and digital, in the learning process as in Arzarello and Robutti (2010). In fact, outside the field of mathematics education, a major argument for using digital resources is the difficulty of accessing the original object or phenomenon (Bétrancourt 2005). While existing studies focus on the use of a digital artifact which simulates an existing and accessible physical artifact, such as the virtual abacus in Poisard et al. (2011), neither the coherence between the uses of the two artifacts, nor the design of the digital artifact given the physical one, is questioned. Moreover, physical and digital resources which address the same mathematical content do not seem to belong to the same system of resources for teachers (Gueudet and Trouche 2009).

The aim of our work is hence to study the means by which the use of technology adds value to the use of other kinds of teaching equipment in the classroom, in particular physical objects. Our idea is to provide the students with a "duo of artifacts", that is, a physical pedagogical artifact and its digital counterpart. Our hypothesis is that, under certain conditions, such a duo of artifacts can enlarge and improve the learning experience of the students. The two artifacts must have some common characteristics, enabling transfer and reinvestment from one to the other. However, as learning is intended, differences between the two are also required to foster the evolution of student knowledge. Similarities and differences between the two artifacts are intended to produce continuities and discontinuities in the use of the artifacts by the students.

We have accordingly designed a digital artifact corresponding to a given physical artifact, based on an analysis of the possible and relevant elements of similarity as well as the necessary and useful elements of rupture in the duo of artifacts. More precisely, we have

\footnotetext{
${ }^{1}$ http://www.unige.ch/math/EnsMath/Rome2008/WG4/WG4.html. Accessed 30 July 2013.
} 
chosen an existing physical pedagogical artifact, the pascaline, ${ }^{2}$, for which the learning potentialities have already been established (Maschietto and Ferri 2007; Canalini Corpacci and Maschietto 2011, 2012) and we have designed a digital counterpart, the e-pascaline, using Cabri Elem technology $\mathrm{y}^{3}$.

In this contribution, our purpose is to present the design of the e-pascaline and the way in which it was informed by the theoretical analysis of the physical artifact together with its use by students in several teaching experiments. We will discuss some characteristics of the resulting duo of artifacts, in terms of continuity and discontinuity, and ways in which the duo can be implemented in pedagogical scenarios.

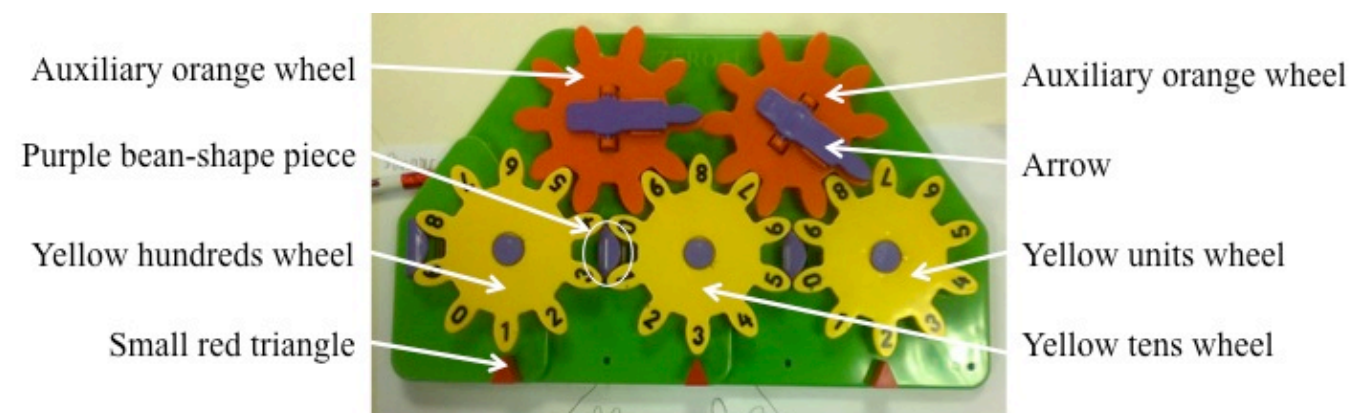

Figure 1. The physical pascaline

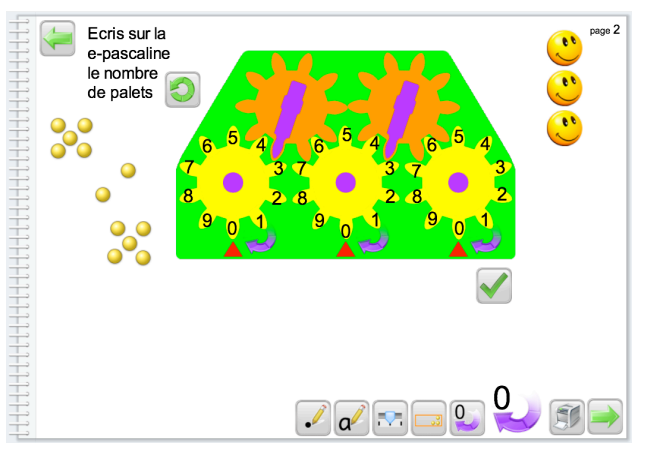

Figure 2. The e-pascaline in the page of an e-book.

In the first part of this paper, we explore existing work about the design of digital resources based on physical objects. The second part presents two theoretical frame- works, instrumental genesis and semiotic mediation, that enable an understanding of the connection between the two artifacts. In Sect. 3, we present studies about the use of the physical pascaline that have informed the key elements of the e-pascaline design. In Sect. 4, we present the e-books including the e-pascaline.

\footnotetext{
${ }^{2}$ Its commercial name is Zero +1 , produced by Quercetti Company.

${ }^{3}$ Cabri Elem technology is produced by Cabrilog Company.
} 


\section{The design of a digital learning artifact: example of interactive animations and virtual simulations}

Several research papers have considered the design and study of the use of digital/virtual tools that have some relationship to material tools.

Concerning research dealing with digital technologies, one major trend consists in designing interactive animations that aim to model the functioning of existing objects or phenomena. Schnotz and Lowe (2003) studied the learning potential of such animations and argue that education should focus on the semiotic and sensory levels of multimedia rather than on the technical level. They highlight the importance of taking into account the complex interactions between external representations (texts, graphics, etc.) and internal (mental) representations constructed by learners. They recommend educators to focus on a better understanding of the processing demands associated with different kinds of representation and their function in learning, in order to discuss the widespread assumption that rich multimedia environments always result in extensive cognitive processing. Boucheix (2008) states that interactive animations are not always efficient for learning and may even be a drawback for student understanding of dynamic processes. In particular, he points out that user control of the animation is key but can lead the user to focus on the more salient perceptive phenomena and not on less visible but more relevant facts. A similar conclusion may be reached in the case of dynamic geometry in mathematics learning. Some very popular dynamic geometry diagrams consist of a figure that stu- dents transform simply by dragging some pre-determined points or by dragging cursors (this kind of task can be summarized as "move this point and observe"). Students explore such diagrams without any requirement to anticipate their behavior, which is not very efficient for learning (Bétrancourt 2005). In fact, in other kinds of dynamic geometry tasks, when students are free to explore dia- grams, they do not easily move points and they focus on irrelevant spatial properties of the figure. We have shown that students have to learn how to use the drag mode with a mathematical purpose (Soury-Lavergne 2006).

Thus, as far as animation is concerned, our digital artifact should offer several representations in interaction in order to provide a rich learning environment. Furthermore, the technical aspects of the animation are not as important as the intended activity of the learner on the sensory-motor and semiotic level. In this regard, Bétrancourt (2005) has differentiated two kinds of interactivity in animations: control, or interactive behavior as in simulations. 
Simulations are also present in mathematics education. The availability of different kinds of software (from dynamic geometry software to professional software for virtual reality) enables simulations of physical tools to be constructed. There are a variety of such tools: tools from the history of mathematics that were described and constructed, or sometimes only described, in mathematical treatises, and also modern tools. Several websites present different kinds of resources, such as interactive simulations, in which the user can change the initial configuration or vary the value of some parameters, or watch video-recordings showing the functioning of the tools, etc. We will consider two examples.

The first example concerns the resources available on the website ${ }^{4}$ ix-quadrat of Zentrums Mathematik in Munich. In the Interactive section, a simulation of the Addiator Duplex is proposed: the front of the tool is reproduced, and the instructions to do addition/subtraction are written on the right. It is a very interesting simulation for lovers of arithmetical devices. By following the instructions, it is possible to use this tool in the same way as the physical tool; even the pencil is represented. How- ever, from an educational point of view, there are no didactical questions or suggestions for use addressed to the students. A similar virtual environment exists for the pas- caline of Blaise Pascal, developed by C. Bascoul ${ }^{5}$ at the Institut Français de Mécanique Avancée in Clermont-Ferrand. From these examples, it appears important that the simulation is presented to the student with a question or a problem to solve and not only the demand to understand the functioning of the machine.

The second example concerns mathematical machines (Maschietto 2005) which are reconstructions of historical tools for perspective drawing, geometrical transformations, and drawing curves (the pascaline can be considered as a mathematical machine for arithmetic). On the website ${ }^{6}$, each mathematical machine has at least a digital version, made with dynamic geometry software or a Java applica- tion. Following Schnotz and Lowe's analysis (2003), Maschietto et al. (2004) studied how students can make sense of animations of mathematical machines in a teaching experiment about perspective in primary school. At the end of teaching sessions in which the students worked with machines for perspective and historical drawings, animations showing the use of those machines (consisting of video without the possibility of interaction) were watched by students. The analysis of students' worksheets is consistent with the position that the use of

\footnotetext{
${ }^{4} \mathrm{http} / / /$ www-m10.ma.tum.de/ix-quadrat/. Accessed 30 July 2013.

${ }^{5} \mathrm{http}: / /$ christophe.bascoul.free.fr/pascaline_dossier/web.html. Accessed 30 July 2013.

${ }^{6} \mathrm{http} / / /$ www.macchinematematiche.org/. Accessed 30 July 2013.
} 
animations in teaching and learning mathematics increases learning if the construction of mathematical meaning implicated in animations is expressly supported.

Studies about relationships between the manipulation of physical and digital objects have been carried out by Manches et al. (2010) in number problem solving (in particular, partitioning tasks with 4-8-year-old children), following the theory of Physically Distributed Learning (Martin and Schwartz 2005). They highlight that under- standing this relationship is not only relevant in choosing which materials are made available to students but also in designing new materials.

Manches et al. proposed tasks in three different con- texts: with no materials; in a physical context; or in a virtual context. Constraints on action were given either by oral instruction or as features of the context. Depending on the constraint conditions of the contexts, pupils' strategies were very different and were related to their possible actions. For instance, the commutative strategy appeared in the physical context because it was possible to group and grasp several objects and move all of them at the same time. This strategy was abandoned in the digital context, however, because of the constraint of moving one object at a time and the requirement for fine motor control skills in using a mouse. In addition, in the physical context, counting was supported by being able to touch objects and also by the spatial organization of objects on the table (a larger space than a screen). In conclusion, "interactive technologies can be designed to foster or constrain these actions and the challenge is to identify which actions are most appropriate for learning in different tasks" (Manches et al. 2010, p. 639). Although this study does not demonstrate what kind of context is preferable, it shows that "different interfaces can influence the actions children make in a numerical task. If different actions lead to different ideas, it is important to consider how a particular interface can foster the desired ideas we want children to grasp" (ibid., p. 639).

In conclusion, we will not focus on the fidelity of the digital artifact to the technical features or the appearance of the physical object, but rather on its potential for learning. We do not need an animation, in which users have a restricted choice of interaction strategies (the control type of interaction according to Bétrancourt, such as dragging a cursor to see some states of the machine), but rather a simulation, which offers a range of interaction strategies and which is controlled by the user. Moreover, every component and behavior of the material machine will not necessarily be simulated; only the characteristics supporting mathematical meanings need to be implemented. 
More importantly, even if the relevant characteristics are implemented in the simulation, it does not inevitably follow that the user will focus on these characteristics while working. According to the authors quoted above, the use of the digital artifact — considered as a rich multimedia environment - has to be organized within a specific didactical methodology, mainly in association with problem solving.

\section{Research questions}

The aim of this paper is to develop the idea of a duo of artifacts, with respect to the pascaline and e-pascaline. According to this idea, the material and digital artifacts are not considered as in a sequence in time, in which the use of the material artifact precedes the use of the digital one, or as in a design project whose aim is to construct and only use the digital counterpart of the material object. We intend to study the duo of artifacts to verify if the use of one artifact adds value to the use of the other. In this case, two different kinds of questions can arise. First, if the two artifacts already exist, questions are related to the kind of scenario to be constructed in order to support the added value in the use of the duo with respect to student learning. Second, if a process of design is foreseen, as in this paper, the research questions are as follows: (1) what are the possible choices of features for the digital counterpart with the aim of constituting a duo in which each artifact would add value to the use of the other; and (2) what theoretical elements can support the analysis of the material artifact and the choice for the design of the digital one?

\section{Theoretical perspective on (dis)continuity in a duo of artifacts}

The instrumental approach and the theory of semiotic mediation are the main theories used to inform the design of the e-pascaline in order to favor an articulated use of both artifacts in the learning process.

The study of the duo of artifacts, as specified above, is based on the theoretical frameworks of the instrumental approach and the theory of semiotic mediation, in line with research on material (Bartolini Bussi and Mariotti 2008) and digital artifacts (Drijvers et al. 2010). More precisely, the two theoretical components are used in three different steps of our methodology. First, they provide the frame- work for studying the existing physical pascaline and the way in which its use results in student mathematical learning. Secondly, the main point of this paper, they inform the design of the e-pascaline, in order to specify and improve the articulation between physical and digital artifact use, rather than just 
Postprint version

continuity between the two artifacts. Finally, although beyond the scope of the present paper, they will be used to study the learning processes of students using both artifacts.

\subsection{The instrumental approach}

The instrumental approach (Vérillon and Rabardel 1995) emphasizes the distinction between an artifact (a material or abstract object, already produced by human activity) and an instrument (a mixed entity with an artifact component and a cognitive component, represented by utilization schemes). This approach may be used to describe and understand the process by which an available artifact becomes an instrument in the hand of the user to perform a particular task. This process is called instrumental genesis, which is the key concept of the instrumental approach. Two different processes are identified: instrumentation (concerning the development of utilization schemes by the user) and instrumentalization (concerning the emergence of artifact components and their modifications for the user's purposes). From an educational point of view, when a student has to use an artifact to perform a task proposed by the teacher, he initiates his personal instrumental genesis related to that artifact. Utilization schemes, developed during activities, are of particular interest because they are strongly related to the construction of knowledge (Vergnaud 2009; Trouche and Drijvers 2010) that is the aim of teachers' work. In consequence, the instrumental approach is a means to foresee the learning possibilities of a given artifact.

Moreover, given one artifact, diverse instrumental geneses can occur, leading to a diversity of instruments. These depend both on the subject and on the associated tasks. For instance, Restrepo (2008) studied the case of instrumental genesis of the drag mode in the use of dynamic geometry environments for the learning of mathematics. She identified several possible instruments and observed their appearance in student activities over a school year. The tasks given to the students were chosen in order to favor some of the possible instrumental geneses and the construction of some instruments instead of others. Therefore, the tasks for which the artifact is used are important elements to take into account when studying the use of an artifact with a learning aim.

The artifact itself may refer to a diversity of material or symbolic objects. In Restrepo's work, the artifact considered was a specific functionality of a technological environment. In our study, one artifact is the pascaline, a physical mathematical machine (Fig. 1). The other part of the duo is the e-pascaline, which appears as a component in e-books designed with Cabri Elem technology (Fig. 2). The e-book is an interactive software, in which the 
user manipulates the e-pascaline to solve problems. The precise description of the pascaline, the e-pascaline, and the e-books is given in Sects. 3 and 4.

Rabardel and Bourmaud (2003) state that the new tools and functionalities are not the result of an isolated instrumental genesis. The new instrument forms a system that incorporates previous ones. The usages of the two artifacts, the pascaline and the epascaline included in the e-books, will interact and, as a consequence, the two instrumental geneses will intertwine. Because our aim is to design the e-pascaline to enhance and enlarge the learning experience of the students and not to produce the most realistic machine, we will ground our design choices in the results of instrumental analysis. In particular, the research question (2) about the theoretical elements to support the analysis can be stated as follows: (2a) regarding the instrumentation processes, which schemes emerging in the use of the physical pascaline should be transferable to the use of the epascaline, that is, supporting continuity between the two artifacts, and which ones should be pre- vented; and ( $2 b$ ) regarding the instrumentalization processes, which elements of the physical pascaline that emerge during the students' use should be transferred and represented in the e-pascaline, supporting continuity, and which ones should be ignored?

\subsection{Artifacts and semiotic mediation}

According to Sáenz-Ludlow and Presmeg (2006), mathematics and the teaching of mathematics are essentially symbolic practices in which signs are invented, used or recreated in order to facilitate cognitive operations. In this paper, we consider the theory of semiotic mediation (Bartolini Bussi and Mariotti 2008) as a theoretical framework for the use of tools in mathematics education. The theory of semiotic mediation is grounded on the one hand within a Vygotskian perspective in the role of artifacts in human activity and, on the other hand, in the idea that cultural artifacts can be considered as embedding mathematical knowledge, emerging when the artifact is used to accomplish tasks. The aim is to describe and explain the process starting with the student's use of an artifact and leading to the student's construction of particular mathematical content.

The design of a teaching sequence which integrates the use of an artifact is based on the analysis of the semiotic potential of the artifact and on the planning of different kinds of activity. Concerning the former, the relationships between specific mathematical content and the use of an artifact to accomplish a task can be seen from two points of view: that of the student using the artifact and constructing personal and situated knowledge; and that of the expert, the teacher, identifying mathematical content in that use. This double semiotic 
link is the semiotic potential of that artifact. Following Mariotti (2012): "Outlining the semiotic potential of an artifact requires a fine grain analysis of the artifact and its use, analysis that encompasses multiple perspectives, including the epistemological and the cognitive perspective, but also a historic and a didactic perspective. The richer the description of the semiotic potential the richer and more powerful is the base for the design of an instruction sequence centered on exploiting such an artifact as a tool of semiotic mediation" (p. 30). The instrumental approach "with the notion of utilization scheme, suggests very effective tools of analysis for out-lining the semiotic potential of an artifact" (p. 31). This kind of analysis allows the teachers to identify which tasks it is possible to propose to students and how to propose these tasks, so that the tasks can be understood by the students who are involved in the resolution process.

The essential elements of the analysis of the semiotic potential of the pascaline will be presented in the next section. These analyses were used for teaching experiments carried out in Italian and French primary school classes. According to the definition, the analysis of the semiotic potential of an artifact is enriched by the analysis of these teaching experiments, in particular the component related to students' personal meaning. For the design of the e-pascaline, the research question (2) is specified as follows: (2c) which signs emerging during student use of the pascaline should be used to design the e-pascaline; and (2d) what elements should be taken into account in order to define the semiotic potential of the e-pascaline, if we consider it as a new artifact?

The two theories will frame the analysis of student use of the pascaline and will determine the characteristics of the e-pascaline. We are going to focus on the main pascaline schemes of utilization and choose which of these should be transferable to the e-pascaline, according to their semiotic mediation potential. In the e-pascaline, we will also implement only the features of the pascaline which have semiotic potential for the mathematical knowledge to be learned.

\section{The physical pascaline}

\subsection{The arithmetical machine}

The pascaline is a small plastic tool (Fig. 1) with a gear train of five wheels. This machine allows numbers to be written in the decimal position system and arithmetical operations to be performed. It is inspired by the mechanical calculator designed by Blaise Pascal (1623$1662)$ in 1642. 
The lower wheels of the pascaline (Fig. 1) display digits from 0 to 9 and represent units (lower right wheel), tens (lower middle wheel), and hundreds (lower left wheel). The upper wheels are auxiliary wheels to transmit motion (by the means of the arrows on them). Each wheel of the pascaline can rotate clockwise and anti-clockwise. When the units wheel has turned a complete rotation clockwise, the right upper wheel makes the tens wheel go one step forward. The same is true for the tens wheel and the hundreds wheel. The purple beanshaped piece connected to the left of each lower wheel forces a jerky motion on these wheels. The representation of the number is read above the small triangles under the lower wheels.

Two procedures can be performed to write numbers, starting from the 000 position above the small triangles: iteration (repeating the operation of pushing the units wheel on the right one step clockwise until the number to represent is reached) and decomposition (separately pushing each wheel one step at a time, until the number of units, tens, and hundreds is reached), which is based on the decimal position system.

Concerning arithmetic operations, for instance addition, two procedures can be performed: addition by iteration (the first term is represented, the second one is added by pushing the units wheel on the right as many steps clock- wise as will represent the number to add) and addition by decomposition (the first term is represented, the second one is decomposed into units, tens, and hundreds, and each of them is added to the corresponding wheel by clockwise iteration). At the end, the result is shown above the small triangles. In each of the two procedures, the user's actions are different for the first and the second terms: the user represents the first term on the pascaline and can read it above the triangles, then the user turns the wheel according to the second term, which is never displayed on the pascaline. To summarize, the first term is represented and the second is performed.

For the operation of subtraction, the difference from addition is the anti-clockwise direction of the lower wheels' rotation. The two other operations, multiplication and division, are made by repeated addition and subtraction operations.

\subsection{Feed forward from students' uses of the physical pascaline for the design of the e-pascaline}

Within the two theoretical frameworks presented above, some teaching experiments have been carried out with the physical pascaline in primary schools (Canalini Corpacci and Maschietto 2011, 2012; Casarini and Clementi 2010; Maschietto 2011; Maschietto and 
Ferri 2007; the Mallette Project ${ }^{7}$ ). In this section, we present some elements from the analysis of these teaching experiments that have been useful in our choices for the design of the e-pascaline.

The analysis of students' activities pays attention to instrumental genesis and the emergence of procedures to both represent numbers and perform arithmetical operations. According to the analysis of semiotic potential, the two procedures are strictly related to students' knowledge of place value. In particular, this knowledge is essential in decomposition procedures.

During the students' first activity, exploration of the pascaline in terms of its structure and components, relationships between components (gears train), and the movement of rotation (that we call the "functioning principle") were observed. The manipulation of the pascaline includes different gestures, called in general "gestures of usage". For instance, some students turned the auxiliary upper wheels, gripping the purple arrows upon them (arrow gesture of usage, Fig. 3 on the left) as a knob or a small lever; lower wheels were also gripped by hand and turned (wheel gesture of usage, Fig. 3 in the middle). The use of fingers (in particular the forefinger) emerges afterwards (one-tooth-at-a-time gesture of usage, Fig. 3 on the right).

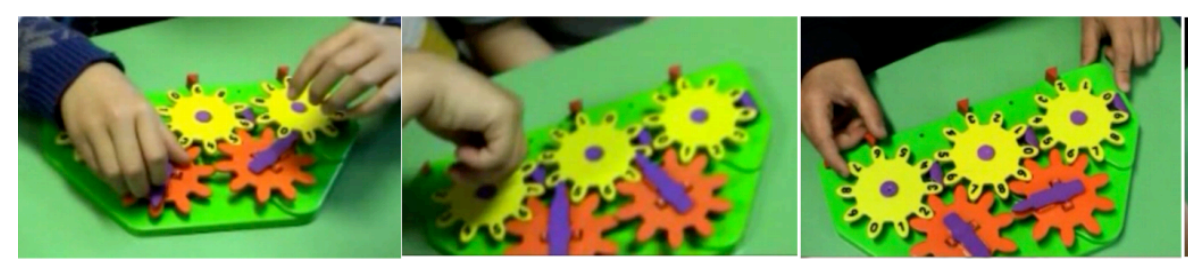

Figure 3. Three examples of students' gestures of usage (grade 3). On the left the arrow gesture of usage, in the middle the wheel gesture of usage and on the right, the one-tooth-at-a-time gesture of usage.

Some of the identified gestures should be reinforced by tasks in the e-pascaline e-book, while other gestures do not have to be reproduced. For instance, the arrow gesture of usage could be an obstacle when verbalizing the utilization schemes for addition, because the orange and the yellow wheels rotate in opposite directions. With respect to this feature, there should be a discontinuity.

Initially, two crucial elements needed to be discovered by the students: the way in which the rotation of one wheel leads to the movement of another wheel; and the relationships

\footnotetext{
${ }^{7}$ A French project supported by the Ministry of Education, directed by the IFE'; a part of the project concerns the use of the physical pascaline and the design of the associated e-books. http://educmath.ens-lyon.fr/Educmath/recherche/equipes-associees/mallette. Accessed 30 July 2013.
} 
between the lower wheels and the red triangles, which point to teeth with digits. The first element corresponds to the mathematical meaning of "number to be carried", while the second one is a fundamental component of the utilization scheme for reading number. The students also found that the movement of the wheel is not continuous, but jerky. On the basis of these elements, the rotation of a tooth at a time (a discrete movement of wheels) is a fundamental feature of the pascaline that has to be kept in the e-pascaline, as an element of continuity. It is related to the mathematical meaning of '?1' (or ' -1 ', depending on the direction of rotation).

According to the theory of semiotic mediation, after the exploration of the pascaline, students were invited to describe the pascaline and its functioning, using drawings. This kind of activity solicits the production of personal signs. Some signs ("accounts" in Trouche 2004, p. 296) in situated texts can be interpreted in terms of the visible part of utilization schemes. Students' drawings show that some gestures and movements pass to the graphical medium. For instance (Fig. 4), signs can replace (1) gestural action arising from the activity with the artifact (hand for a gesture of usage), (2) part of the utilization scheme to perform addition (arrow with +6 , although the rotation is wrong, Figs. 4 and 5), and (3) the movement of a wheel (small stroke around the wheel on the right) as result of that action (arrow).

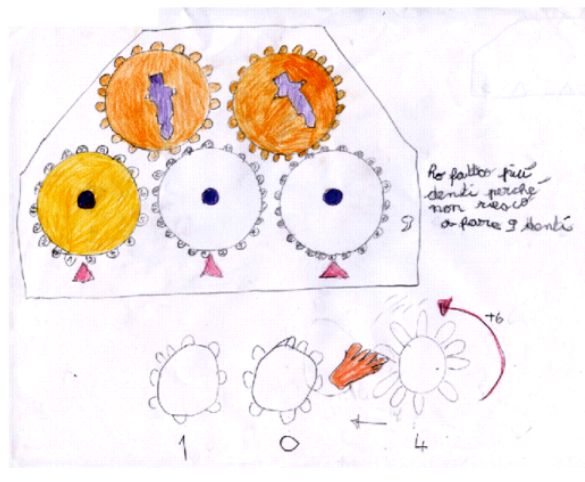

Figure 4. Student's description of the pascaline, with the representation of a hand and fingers (in Italy, grade 3 , nine year old students).

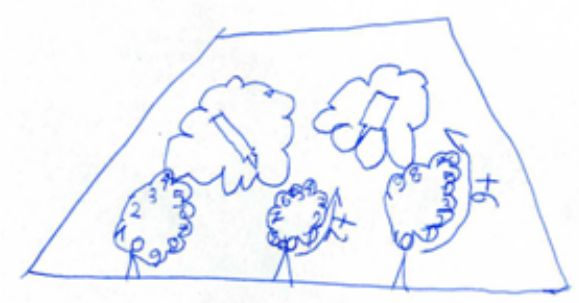

Figure 5. Student's drawing of the pascaline, explaining how to write the number 26 and wheel gesture of usage of the pascaline (in France, grade 2, seven year old students). 
As a consequence, the design of the e-pascaline should take into account the presence of arrows referring to the movement of the wheels during the use of the pascaline.

Students' personal systems of instruments (Trouche 2004) also emerge during tasks with the pascaline. The use of the physical pascaline is embedded in a complex system of instruments that should be taken into account in the digital artifact. For instance, for performing multiplication and division, students use paper and pencil or their hands in order to keep track of repeated additions and subtractions. The e-pascaline should offer this kind of tool to students.

Other characteristics and components of the pascaline are not necessary for the design of the e-pascaline. The bean-shaped piece is not used by the students to perform tasks with the pascaline, and they do not always reproduce it in their drawings (it appears in only 22 out of 33 drawings made by 6-year-old students, in 3 out of 20 drawings made by 7 -yearold students, and in 2 out of 6 drawings made by 8 -year-old students in the French experiments). No usage schemes are developed and, because no semiotic potential is identified, the decision was made not to reproduce it in the e-pascaline. This is not the case for the arrow on the upper wheels, which is sometimes used by students and appears in a majority of drawings (it appears in every drawing made by 6-year-old students, in 11 out of 20 drawings made by 7-year-old students, and in 3 out of 6 drawings made by 8 -year-old students in the French experiments). This arrow must be represented in the e-pascaline, but with no possible action, exactly like the upper wheels.

Finally, the sounds produced when the wheels turn could represent continuity or discontinuity. The choice is strictly related to didactical use of the duo of artifacts by teachers. Therefore, it could become a parameter of the e-pascaline.

The analysis of the teaching experiments with the pascaline shows several elements to be taken into account from the perspective of the duo of artifacts and continuity/ discontinuity between them. An important element is the emergence of gestures and their relationship to mathematical meanings: from this point of view, some gestures are relevant, while others are not. This means that a choice must be made about which gestures to support in the work with the e-pascaline, that is, the gestures which are mathematically significant. In consequence, corresponding elements in the pascaline will be animated in the e-pascaline. Another element concerns the graphical representations of user actions in students' drawings. Some, such as arrows for rotation, can be used to support the instrumental genesis of the e-pascaline. In so doing, we are aware that the signs in students' drawings 
Postprint version

after using the pascaline are the condensation of actions, while the signs in a digital environment suggest actions to perform.

\section{Design of the e-pascaline and the e-book}

\subsection{The environment for the design of the e-pascaline}

The e-pascaline has been created within the authoring environment Cabri Elem Creator. It appears as an entity displayed on the pages of Cabri Elem e-books. Once an e-book has been created in the authoring environment, it can be used directly by teachers and students in the more restricted environment of Cabri Elem Player (for more details about the design of Cabri Elem e-books, see Laborde and Laborde 2011 or Mackrell et al. 2013).

The e-pascaline has been created as a complex object that looks like the physical object. The shapes, the main constitutive elements, and the colors are respected (see Fig. 1). The implementation of the e-pascaline has required additional design choices, however, as the e-pascaline is not a simple replica of the physical one. The choices and their consequences can be related to two types of affordances and constraints: the authoring environment and the didactical choices.

The authoring tool is a mathematical environment based on direct manipulation. Images of real-world objects may be used, with some behaviors added. With these possibilities and constraints, the key behaviors of the wheels have been implemented in the e-pascaline: the jerky rotation of the wheels and the automatic driving of the wheels under certain conditions. There are many differences between the two artifacts, however. We detail now the common points and the differences that have consequences for its usage by the students.

\subsection{Continuity and discontinuity}

A first difference between the pascaline and the e-pascaline results from our decision to not allow direct manipulation of the wheels of the e-pascaline. This could lead to a continuous movement of the wheel, as in the existing usage scheme with the pascaline. Instead, the epascaline wheels turn by the use of buttons that launch their animation, with two buttons for each wheel in order to turn the wheel in either direction. In consequence, the wheel gesture of usage is not possible any more, creating a discontinuity in the instrumentation from the pascaline to the e-pascaline. Another discontinuity concerning these buttons results from the fact that they are displayed only when the signification of the wheels' 
movements can be mathematically interpreted by an operation in the non-negative integers. It means that when the e-pascaline is reset, the three buttons to move anti-clockwise are hidden, or, for instance if the e-pascaline is under 10, the buttons to move the hundreds and tens wheels are hidden. This choice results from the semiotic analysis, pointing out the delicate didactical question of giving a mathematical meaning to 000 minus 1 .
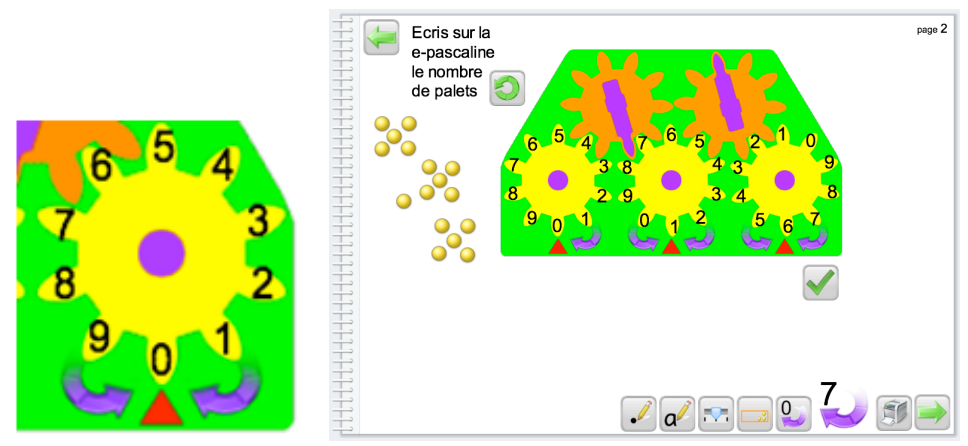

Figure 6. The two curved arrows below each wheel are the buttons that launch the rotation of the e-pascaline wheels. In the screen shot of the entire page, there is only one curved arrow under the hundreds wheel because 100 cannot be subtracted from the displayed number which is 16 .

Moreover, the choice of arrows to represent the wheel action buttons was guided by our semiotic analysis. The arrows that were signs representing the wheels' movement in the pascaline drawings become a means of action and produce the same movement in the epascaline. This is an example of continuity.

Another design choice concerns the purple arrow on the two upper wheels of the pascaline. In the e-pascaline, the arrow is only a passive image. Therefore, the students' arrow gesture of usage (another usage scheme) is no longer possible. The instrumental genesis of the pascaline that has resulted in the emergence of the purple arrow (instrumentalization process) as a means to rotate the wheel (instrumentation process) is interrupted. The actions on the wheels are restricted to the ones that have the intended semiotic potential for the learning of mathematics (i.e., $+1,-1,+10,-10,+100$, and -100 ).

A final example of design choices is the initialization or resetting of the e-pascaline. Using the pascaline requires regular resetting of the wheel positions to 000 by acting on each wheel. In the e-pascaline, this is obtained by clicking on the purple circle in the middle of each wheel or clicking on a reload button (Sect. 5.3).

The e-pascaline is embedded in an e-book page, which also provides other components. The "tool box" consists of buttons at the bottom of the page (Figs. 6, 7) which are not part of the e-pascaline but which can be linked to its use. Their presence in the book results from our theoretical analysis of the pascaline utilization. It is possible to pro- vide the 
students with a range of tools that can be freely used while performing tasks with the epascaline. Most of these tools correspond to elements of the system of instruments (in the sense of Rabardel) emerging in the use of the physical pascaline, such as a pen to write with. They are introduced into the e-pascaline environment in the form of a tool to write numbers or text (Fig. 7). The other tools are designed to support the problem solving process and are thus determined more by the tasks than by the e-pascaline. For instance, the cursor marks out a number between 0 and 30 (or 0 and 100) and the user can freely change the displayed number (Fig. 7). This tool may be connected to finger counting. The last tool is a click counter, enabling the student to keep track of the number of teeth that pass in front of the red triangle. The square button resets the click counter.

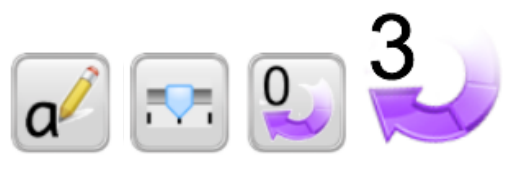

Figure 7. Some tools in the e-book "representing numbers" (from left to right): the writing tool (text and numbers), the cursor display and the click counter reset (the click counter is displayed to the right).

The choice of tools shows clearly that the design of the e-pascaline in the Cabri Elem environment is not reduced to an imitation of the physical pascaline. The e-pascaline environment includes elements that should enrich the learning experience of the student. In fact, Cabri Elem is an environment to design learning activities, including objects and behaviors but also feedback and constraints that characterize the didactical situation.

\subsection{Task design with the e-pascaline in the Cabri Elem environment}

The design of tasks with Cabri Elem opens a large range of possibilities for the use of the e-pascaline. Many different e-books can include the e-pascaline and each e-book may have a number of pages with the possibility of changing every element and behavior in each of them. Thus, design choices can be implemented in some e-books and not in others, according to the learning objectives. This will also enable the evolution of the e-books and their adaptation to the teachers' requirements.

We have elaborated several e-books with the e-pascaline. Here we present two of them: 1 for 6-year-old students about representing numbers, and the other about calculation for 6 or 7 -year-old students. They allow us to show and discuss different ways to exploit the added value given by technology to the use of the physical pascaline.

The learning objective of the e-book about number representation is the decimal system for representing number, more precisely the place value system. The tasks given to the 
students are to represent a number with the e-pascaline, the number randomly determined and dis- played by a collection of counters or by an oral message.

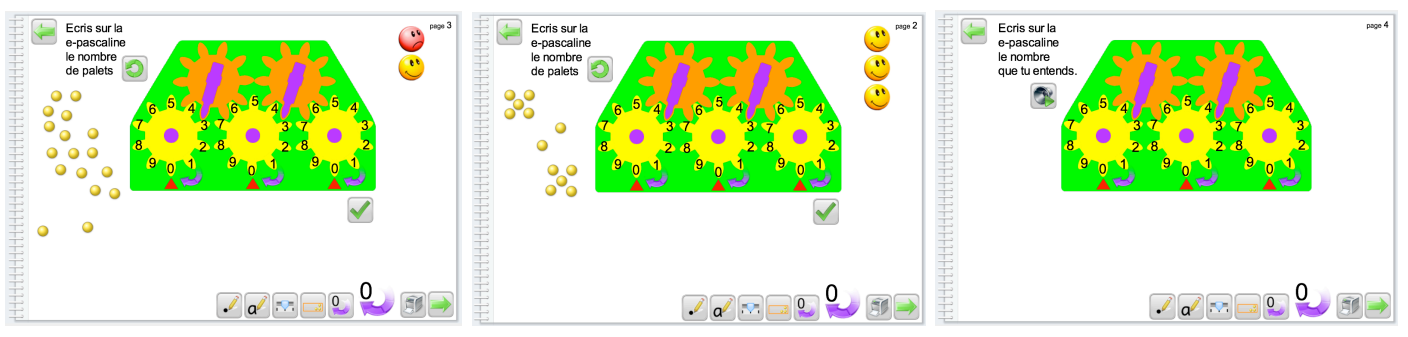

Figure 8. The first and the second page of the e-book "representing numbers" show the smileys obtained as evaluation feedback.

On every page, the objects are almost the same (Fig. 8): the e-pascaline, a reset button (to get a new number to be represented), a set of tools, an evaluation button (to get feedback about the correctness of the number represented by the e-pascaline), and arrows to move to the previous or next page. The visible differences are in the way the target number is displayed: either a collection of tokens with different spatial settings or a speaker to give an oral message. There are other less visible but very important differences, enabling or constraining student strategies.

One of these is feedback, i.e. the reaction of the artefact to the action of the user. We have identified three levels of feedback (Mackrell et al. 2013): (1) direct manipulation feedback, that can be linked to the perceptive aspects of the objects' behavior (for example the rotation of the wheel when either rotation button is clicked, the display of a new collection of tokens when the reload button is clicked, the reset of the e-pascaline wheels to 0 when the purple circle is clicked); (2) evaluation feedback (happy or unhappy smileys are displayed when the student asks if the number represented by the pascaline is correct); and (3) strategy feedback that results from an analysis of student strategy (for example, the fact that the rotation buttons of the units wheel could be hidden after a specific number of clicks on it to force the use of the decomposition strategy).

The other important elements of the pages are some parameters (didactic variables), with values chosen in order to facilitate or to prevent particular strategies. The two main didactic variables of this e-book are: (1) the size of the number to write-below 30, from 30 to 69 , or from 70 to 99 (based on the French irregular representation of numbers from 0 to 16 and from 70 to 99); and (2) the way the target number is given, not using the standard place value notation - counters up to 30 (Fig. 8 on the left), or an oral message that can be repeated as many times as needed (Fig. 8 on the right). 
In every page, the two main procedures to represent a number on the e-pascaline, as with the pascaline, are iteration, that is, the rotation of the units wheel according to the target number, or the decomposition procedure, using the decimal representation of the target number. This second procedure is possible only for students who can anticipate the representation of a number using digits, eventually writing it down, and displaying it with the e-pascaline. Other possible strategies are matching every counter with a click on the right arrow of the units wheel and stopping as soon as every counter has been enumerated, which avoids the use of number, and matching the rotation steps of the unit wheel with the counting rhyme. Note that the difficulty of the task should not be in finding the number of counters; students may use the pen to mark each enumerated counter or the ten-counters box (which enables counters to be grouped in tens, Fig. 9) to easily determine the number of counters in the collection.

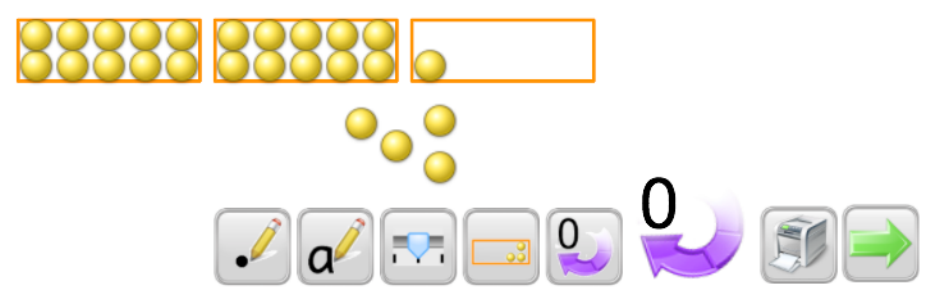

Figure 9. The ten-counters box helps students to find out the number of counters.

In this example, the added value lies not only in the e-pascaline functioning but also in the task: the way in which the task is presented (by displaying counters, or giving the oral name of the numbers), the possibility of repeating the task with a different number as often as wanted and at the pace of each student, and the immediate and personalized feedback which is crucial for student involvement in the task.

In the second e-book we present in this paper, the added value lies in a deeper connection between the e-pascaline and the task. The e-book is about "minimizing the number of clicks" to write a number on the e-pascaline. There are various procedures to represent a given number on the e-pascaline starting from 000. Each of the procedures asks for a given number of clicks on the six curved arrows. Given the number $\mathrm{N}=\mathrm{h} \times 10^{2}+\mathrm{t} \times 10+\mathrm{u}$, the iteration procedure asks for $\mathrm{N}$ clicks and the decomposition procedure asks for $\mathrm{h}+\mathrm{t}+\mathrm{u}$ clicks. Other procedures may require even fewer clicks, however. For example, 17 is equal to 20 - 3 and therefore can be represented with only 5 clicks on the e-pascaline. With the physical pascaline, the task would be difficult to implement with young students because it requires two simultaneous processes: representing the number and counting the clicks. The e-pascaline counts the clicks automatically and gives two kinds of evaluation feedback: a 
message "17 in 8 clicks" or the same message with a smiley when the minimum is reached (Fig. 10). The e-pascaline could also hide the arrows as soon as the minimum number of clicks has been reached, forcing the student to stop and restart with a different procedure. The effect of this strategy feedback on the evolution of student strategy is yet to be studied, however.

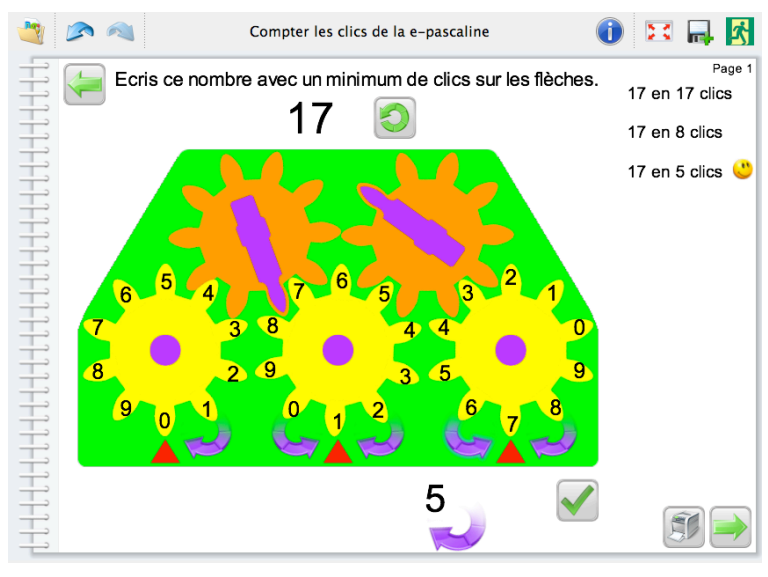

Figure 10. E-book "minimizing the number of clicks" with target number 17 and three evaluations shown on the right. The smiley is associated with message 17 in 5 clicks.

This task requires students to explore different ways of reaching a number through combinations of addition and subtraction. It also requires the student to record the procedure in order to remember it and to share it with other students and with the teacher. Therefore, a careful organization of the task, including the e-book and the recording of procedure, is required.

\subsection{Conclusion about the added value of the e-pascaline and e-books}

In e-books described above, the added value of technology lies in the e-pascaline, the associated tools, and the proposed tasks.

First, the added value comes from new features of the e-pascaline, such as the facility to reset, to count the number of clicks, and to operate the e-pascaline by the use of six distinct action buttons. It comes also from preventing some student actions for which the semiotic potential would lead beyond primary school mathematics, such as subtracting a number from 000 or using the arrows on the upper wheels to turn the wheels. Further evolution of the e-pascaline features are planned: for example, adding supplementary wheels to deal with thousands; or hiding parts of the e-pascaline, such as the upper wheels or the lower wheels apart from the tooth in front of the triangle (as in Pascal's original machine). 
Second, the added value comes from additional tools, in particular those that give feedback, a key feature of the e-books. Feedback is more frequent and adapted to each pupil action and procedure than is possible with the pascaline alone. In particular, evaluation feedback is independent from teacher evaluation, which corresponds to a more general characteristic of technology. Additional tools also facilitate parts of the task which are not central (such as containers to group counters by tens).

Finally, the added value of technology comes from the careful design of the tasks. The tasks are embedded into the e-books. They are defined by a choice of values for didactical variables and by feedback, especially strategy feedback. The utility of the e-pascaline and associated tools for learning depends on the proposed tasks. Indeed, the main added value of the digital environment lies in the possibility of creating tasks and adding constraints and feedback to the use of the e-pascaline.

We should also mention the advantage of being able to display an e-pascaline on a projector, enabling the entire class to share procedures during collective activities. This is not possible with the pascaline.

However, it should be noted that the pascaline also has added value compared with the epascaline, which explains why one cannot be substituted for the other. First, kinesthetic feedback is given by the pascaline and not by the e-pascaline, and this kinesthetic feedback is different when the tens or the hundreds change. Second, feedback from sound is also given when wheels rotate and is different when several wheels turn together.

\section{Conclusion}

In this paper, we have studied how a duo of artifacts can be designed to enhance the mathematical learning experience of students.

Our duo of artifacts is constituted by a physical machine, the pascaline, and its digital counterpart, the e-pascaline embedded into e-books. We have first chosen a physical artifact which studies have shown has relevance for the learning of mathematics. We have then designed the digital e-pascaline, paying particular attention to differences and complementarities between the two artifacts. This paper describes the main elements of the design process, the key features that were taken into account and the theoretical grounding of our choices. The following results guide the further design of duos of artifacts.

Our first research question concerns the possible added value for students' learning provided by the duo of artifacts. A didactical aim has been that students are able first to 
under- stand and explain the mathematical meanings behind their utilization schemes, then to formulate them in a mathematical representation. The e-pascaline and associated ebooks are expected to help students make explicit the epistemic value of their actions by offering them the opportunity to transfer schemes from the pascaline, to improve formulations and procedures, and to vary experiences, which are important elements for learning. Yet, there is still need to investigate in practice the added value of the duo of artifacts.

This added value would result from continuities and discontinuities between the two artifacts. Thus, to design the digital artifact, we needed to determine the features, beyond the graphical and aesthetic aspect of the objects, that should be continuous or discontinuous.

Our second research question concerns the theoretical elements and the analysis of the physical artifact that can inform the choice of continuities and discontinuities.

With regard to the instrumentation process (question 2a), utilization schemes should be major elements of continuity in the use of the two artifacts. However, some actions and utilization schemes are not related to a mathematical meaning (question 2d) and hence should no longer be possible with the digital artifact. Thus, only the features that support the transfer of schemes with semiotic potential have been selected and implemented into the digital artifact.

With regard to the instrumentalization process (question $2 b$ ), not all components of the physical artifact are instrumentalized by the students, as student drawings of the physical artifact reveal. This has been a criterion to define the components of the digital artifact. Furthermore, some additional tools emerge in the use of the physical artifact which can be implemented in the digital artifact.

Other components of the digital artifact do not refer to physical components but to signs produced by the students (question 2c). Signs emerging from the use of the physical artifact or from descriptions of user's actions may also be used as means of action in the digital environment and become components of the digital artifact.

With these choices, the use of the two kinds of artifacts by students should foster their access to mathematical meaning. This needs to be validated by experiments, however. Such experiments are planned in the Mallette project, in association with the development of new e-books and teaching scenarios merging the use of the pascaline and the epascaline. The duo of artifacts must also be analyzed from the point of view of teacher appropriation. Our assumption is that providing the teachers with a duo of artifacts should 
help them to better understand the added value of each artifact, in particular the digital artifact, thus supporting their appropriation by teachers.

The design of the duo of artifacts is the first step in our research program that aims to improve the integration of technology into primary school mathematics teaching.

\section{References}

Arzarello, F., \& Robutti, O. (2010). Multimodality in multi-representational environments. ZDM-The International Journal on Mathematics Education, 42, 715-731.

Bartolini Bussi, M.G., \& Mariotti, M.A. (2008). Semiotic mediation in the mathematics classroom: Artifacts and signs after a Vygotskian perspective. In L. English (Ed.), Handbook of international research in mathematics education (2nd ed., pp. 746-783). New York: Routledge.

Bétrancourt, M. (2005). The animation and interactivity principles, in R. E. Mayer (Ed.) Handbook of Multimedia. (pp. 287-296). Cambridge: Cambridge University Press.

Boucheix, J.M. (2008). Young learners'control of technical animations. In R.K. Lowe \& W. Schnotz (Eds.), Learning with animations (pp. 208-234). New-York: Cambridge University Press.

Canalini Corpacci, R., \& Maschietto, M. (2011). Gli artefatti-strumenti e la comprensione della notazione posizionale nella scuola primaria. La 'pascalina' Zero+1 nella classe: genesi strumentale. L'Insegnamento della Matematica e delle Scienze Integrate, 34A(2), 161-188.

Canalini Corpacci, R., \& Maschietto, M. (2012). Gli artefatti-strumenti e la comprensione della notazione posizionale nella scuola primaria. La 'pascalina' Zero+1 e sistema di strumenti per la notazione posizionale. L'Insegnamento della Matematica e delle Scienze Integrate, 35A(1), 33-58.

Casarini, A., \& Clementi, F. (2010). Numeri... in macchina: alla scoperta della pascalina. In USR E-R, ANSAS E-R, Regione Emilia-Romagna \& F. Martignone (Eds.), Scienze e Tecnologie in Emilia-Romagna, (Vol. 2, pp. 141-145). Napoli: Tecnodid Editrice.

Drijvers, P., Kieran, C., \& Mariotti, M. A. (2010). Integrating technology into mathematics education: theoretical perspectives. In C. Hoyles \& J.-B. Lagrange (Eds.), Mathematics Education and TechnologyRethinking the Terrain (pp. 89-132). New York: Springer.

Edwards, L., Radford, L., \& Arzarello, F. (Eds.) (2009). Gestures and Multimodality in the Construction of Mathematical Meaning. Educational Studies in Mathematics, Special issue, 70(2).

Gueudet, G., \& Trouche, L. (2009). Towards new documentation systems for mathematics teachers? Educational Studies in Mathematics, 71, 199-218.

Hoyles, C., \& Lagrange, J-B. (2010). Mathematics Education and Technology-Rethinking the terrain: the 17th ICMI Study. New York: Springer.

Kalénine, S., Pinet, L., \& Gentaz, E. (2011). The visuo-haptic and haptic exploration of geometrical shapes increases their recognition in preschoolers. International Journal of Behavioral Development, 35, 18-26. 


\section{Postprint version}

Laborde, C., \& Laborde, J.-M. (2011). Interactivity in dynamic mathematics environments: what does that mean? Proceedings of ATCM conference. http://atcm.mathandtech.org/EP2011/

invited_papers/3272011_19113.pdf. Accessed 30 July 2013.

Lakoff, G., \& Núñez, R. (2000). Where Mathematics Comes from: How the Embodied Mind Brings Mathematics into Being, New-York: Basic Book.

Mackrell, K., Maschietto, M., \& Soury-Lavergne, S. (2013). The interaction between task design and technology design in creating tasks with Cabri Elem. In C. Margolinas (Ed.), Proceedings of the ICMI Study 22 Conference: Task Design in Mathematics Education, (pp. 81-89). Oxford, UK July 2013.

Manches, A., O'Malley, C., \& Benford, S. (2010). The role of physical representations in solving number problems: a comparison of young children's use of physical and virtual materials, Computer \& Education, 54, 622-640.

Mariotti, M.A. (2012). ICT as opportunities for teaching-learning in a mathematics classroom: the semiotic potential of artifacts. In Tso, T. Y. (Ed.). Proceedings of the 36th Conference of the Intern. Group for the Psychology of Mathematics Education, (Vol. 1, pp. 25-40). Taipei: PME.

Martin, T., \& Schwartz, D. (2005). Physically Distributed Learning: Adapting and Reinterpreting Physical Environments in the Development of Fraction Concepts. Cognitive Science, 29(4), 587-625.

Maschietto, M. (2005). The Laboratory of Mathematical Machines of Modena. Newsletter of the European Mathematical Society, 57, 34-37.

Maschietto, M. (2011). Instrumental geneses in mathematics laboratory. In Ubuz, B. (Ed.), Proc. of the 35th Conference of the Intern. Group for the Psychology of Mathematics Education, (Vol. 3, pp. 121-128). Ankara, Turkey: PME.

Maschietto, M., Bartolini Bussi, M.G., Mariotti, M.A., \& Ferri, F. (2004). Visual representations in the construction of mathematical meanings. Paper for ICME 10 - TSG16: Visualisation in the teaching and learning of mathematics, Copenhagen, Denmark.

Maschietto, M., \& Ferri, F. (2007). Artefacts, schèmes d'utilisation et significations arithmétiques. In J. Szendrei (Ed.), Proceeding of the CIEAEM 59, (pp. 179-183), Hungary: Dobogoko.

Pepin, B., Gueudet, G., \& Trouche, L. (2013) Re-sourcing teachers' work and interactions: a collective perspective on resources, their use and transformation. ZDM - The International Journal on Mathematics Education, 45(7) (this issue).

Poisard, C., Bueno-Ravel, L., \& Gueudet, G. (2011). Comprendre l'intégration de ressources technologiques en mathématiques par des professeurs des écoles. Recherches en didactique des mathématiques, 31(2), 151189.

Rabardel, P., \& Bourmaud, G. (2003). From computer to instrument system: a developmental perspective. Interacting with Computers, 15(5), 665-691.

Restrepo, A. (2008). L'instrumentation du déplacement dans les environnements de géométrie dynamique : le cas de Cabri-Géomètre. Doctoral dissertation. Grenoble: Université Joseph Fourier. 


\section{Postprint version}

Sáenz-Ludlow, A., \& Presmeg, N. (2006). Semiotic perspectives on learning mathematics and communicating mathematically. Guest editorial. Educational Studies in Mathematics, 61, 1-10.

Schnotz, W., \& Lowe, R. (2003). External and internal representations in multimedia learning. Learning and Instruction, 13(2), 117-123.

Soury-Lavergne, S. (2006). Instrumentation du déplacement dans l'initiation au raisonnement déductif avec Cabri-géomètre. In N. Bednarz, \& C. Mary (Eds.), L'enseignement des mathématiques face aux défis de l'école et des communautés, Actes du colloque EMF 2006, Sherbrooke: Université de Sherbrooke.

Trouche, L., \& Drijvers, P. (2010). Handheld technology for mathematics education: flashback into the future. ZDM The International Journal on Mathematics Education, 42(7), 667-681.

Vergnaud, G. (2009). The Theory of Conceptual Fields. Human Development, 52, 83-94.

Vérillon, P., \& Rabardel, P. (1995). Cognition and artifacts: A contribution to the study of thought in relation to instrumented activity. European Journal of Psychology of Education, 10(1), 77-101. 